\title{
Medical Therapy for Fungal Rhinosinusitis
}

\section{${ }^{1}$ Vicky S Khattar, ${ }^{2}$ Bachi T Hathiram}

\author{
${ }^{1}$ Assistant Professor, Department of ENT and Head and Neck Surgery, TN Medical College and BYL Nair Charitable Hospital \\ Mumbai Maharashtra, India \\ ${ }^{2}$ Professor and Head, Department of ENT and Head and Neck Surgery, TN Medical College and BYL Nair Charitable Hospital \\ Mumbai Maharashtra, India
}

Correspondence: Vicky S Khattar, Assistant Professor, Department of ENT and Head and Neck Surgery, TN Medical College and BYL Nair Charitable Hospital, C-7, Swati, Kalyan Complex, Panchmarg, Versova, Mumbai-400061, Maharashtra, India

\begin{abstract}
Medical therapy forms an important modality in the management of fungal rhinosinusitis. This essentially comprises of topical as well as systemic antifungals, steroids or both, depending upon the nature of the disease. The drugs may need to be started preoperatively in some conditions, but it is essentially the postoperative management which is vital to prevent recurrences, and may sometimes continue for months. Discussed here are the various forms of fungal rhinosinusitis and their proposed regimens.
\end{abstract}

Keywords: Amphotericin B, Itraconazole, Voriconazole, Azole, steroids, antifungal.

There are various issues relating to the use of concomitant medical therapy in the management of fungal rhinosinusitis. Firstly, there are different forms of fungal rhinosinusitis, and each requires a specific therapeutic regimen, targeted at the pathogen. Secondly, there are numerous toxicity related issues which need to still be addressed. Thirdly, many newer antifungal drugs are extremely expensive. Lastly and most importantly, there are few randomised controlled trials which may be able to validate the results by various studies.

We shall discuss the treatment modalities for the different forms of fungal rhinosinusitis separately below.

\section{ALLERGIC FUNGAL RHINOSINUSITIS}

It has been shown beyond doubt that surgery for AFRS without postoperative medical management leads to recurrence rates of upto $100 \%$, depending upon the expert referenced. ${ }^{1,2}$

The therapy for AFRS may be local and systemic: Topically, steroid sprays may be used for a minimum period of six months, and gradually tapered off, keeping room for stepping up the dose during an acute exacerbation of this recurrent pathology. Owing to the extramucosal nature of the disease, Amphotericin B washes may also be given, which can take care of the luminal load of the fungus; there is not adequate evidence in literature however, to validate this therapy for AFRS.
The use of oral corticosteroids in the postoperative AFRS patient has followed on the success of its treatment for ABPA. ${ }^{5,7}$ Oral predinosolone can be started in a dose of 0.5 $\mathrm{mg} / \mathrm{kg}$ as a single dose, and may then be tapered off, starting two weeks afterwards, and given for a minimum period of three to six months, with a low maintenance dose being given for even longer periods. Acute exacerbations of symptoms during treatment may be managed with a short step-up of the dose to tide over the crisis, followed by continuation of the earlier maintenance phase afterwards.

Since this is purely a noninvasive entity, the role of systemic antifungal therapy is to be questioned. However, there has been an increased interest in the use of oral itraconazole in a dose of $100 \mathrm{mg}$ to $200 \mathrm{mg}$ twice daily, for variable periods ranging from three weeks to six months postoperatively, probably due to added its anti-inflammatory effect. Studies for ABPA have found an additional clinical benefit from the use of itraconazole, when compared to a placebo. ${ }^{3,4}$ Due to the similarity in the pathogenesis of ABPA and AFRS, this may be worth a thought. Principally however, this appears to be of no use, since AFRS has been defined on the basis of its extramucosal nature; nevertheless the added clinical benefit obtained in ABPA surely asks for further investigation in this field. Nevertheless, Rains et al (2003) have reported retrospectively, that of the 139 patients of allergic fungal sinusitis, treated with steroids and postoperative itraconazole, they found a reduced need for revision surgery. ${ }^{11}$ 


\section{FUNGAL BALL}

An entity, even the surgical treatment of which is so controversial, has an equally controversial debate as regards its medical therapy. Klossek et al (1997) have stated that true fungal sinus balls require no adjuvant local or systemic antimycotic treatment, even if bone destruction is observed. ${ }^{9}$ Following the reports of an immunocompromised patient who developed invasive fungal sinusitis after being operated for a sphenoid fungal sinus ball, Ferguson (2000) has recommended the use of adjunctive systemic antifungal treatment such as itraconazole in immunocompromised patients, especially if there is bone erosion of the involved sinus, while others prefer to keep the patient under close clinical observation in such situations. 8,10

Antibiotics may be used to manage any superadded bacterial infections, along with the regular saline nasal douches and sprays, and even a short course of steroid spray may be administered, as part of the routine postoperative management following functional endoscopic sinus surgery.

\section{INVASIVE FUNGAL SINUSITIS}

The mainstay of management in invasive fungal sinusitis is three-fold:

1. Rapid reversal of the cause of immune-compromise (if any).

2. Radical sinonasal debridement (external or endoscopic) till 'bleeding margins' are obtained, under cover of appropriate.

3. Systemic antifungal therapy.

Systemic antifungal therapy has evolved from the days of empirical intravenous amphotericin, to date, where numerous classes of systemic antifungals are available, which may be deployed, depending on the culture and clinical presentation.

For decades, Amphotericin B was the standard drug for most life-threatening systemic fungal infections. It is associated with numerous toxicities; yet has stood the test of time. It is essentially a lipophilic polyene macrolide, which has an affinity to, and binds with ergosterol (an essential fungal cell membrane component). This causes and increased permeability of the cell membrane, leading to leakage of intracellular components, and eventual cell death. Its lipophilic nature allows it to bind with normal cholesterol seen in mammalian cell membranes, although to a lesser degree, which probably accounts for its toxicity. It also has an immune-modulating activity, which may be achieved by activation of macrophages, which may contribute to the final effect.

- Prior to starting the drug, the patient should be hydrated with $500 \mathrm{ml}$ to $1 \mathrm{l}$ of normal saline. This also supplements the sodium required to maintain the intravascular volume, and inhibit the tubuloglomeurular feedback system, which eventually contributed to a decreased risk of developing nephrotoxicity. ${ }^{12}$

- A test dose should be administered by diluting $1 \mathrm{mg}$ of the drug in 50 to $100 \mathrm{ml}$ of $5 \%$ dextrose, and given slowly intravenously over 20 minutes. ${ }^{13,14}$

- There are two ways to administer the drug: either to start with a low dose, and titrate gradually till a maximal dose is achieved which is bereft of side effects, or to start with a standard dose as per the patient's body weight, and maintain the same till a total permissible cumulative dose may be achieved; there are proponents and opponents of both regimens. Standard doses range between 0.25 to $1.0 \mathrm{mg} / \mathrm{kg} /$ day in a single dose, diluted in $5 \%$ dextrose, to be given slowly intravenously. The bottle and tubing should be protected from sunlight. Maximum daily doses range between $1.2 \mathrm{mg} / \mathrm{kg}$ in adults and $1.5 \mathrm{mg} / \mathrm{kg}$ in children, the latter being reserved for serious invasive mycoses. ${ }^{15}$

- The common side effects are electrolyte disturbances, nephrotoxicity, bone marrow suppression and infusion related reactions. Besides that, headaches, chills and rigors, fever, nausea and malaise may be experienced, which may be managed symptomatically by premedicating with Paracetamol, antihistaminic and antiemetic drugs. Thrombophlebitis can be a troublesome problem, and may be avoided by using a central venous access or alternating peripheral venous lines.

- The patients should be monitored twice weekly for serum electrolytes and renal function tests, and once weekly for a complete hemogram, since the drug may cause bone marrow suppression.

- Patients with previous renal compromise should be monitored more aggressively, even daily, and the dose titrated accordingly. A double dose may also be given on alternate days in such patients. ${ }^{13,14}$

Of late, lipid based formulations of the drug have been made available, by using various lipid carriers. The uniqueness of these formulations is their selective delivery into the Reticuloendothelial syetem (RES), such as the liver 
and spleen, and to lesser degree to the lungs. ${ }^{14,16}$ These drugs are known to have a better side-effect profile as compared to the standard Amphoterecin B deoxycholate. They may be considered in patients who may not be able to tolerate the nephrotoxicity caused by Amphotericin B deoxycholate. ${ }^{15}$ Although their dose-related efficacy may be less as compared to the standard Amphotericin, the fact that higher doses can be used, makes them more efficacious. The doses are as follows: ${ }^{15}$

- Liposomal Amphotericin B - 1 to 5 mg/kg

- Amphotericin B lipid complex - 5 mg/kg

- Amphotericin B cholesterol sulfate complex - 3 to 5 $\mathrm{mg} / \mathrm{kg}$.

Due to problems with correlating CT scans in the early stage of the disease (See article on 'Radiological appearances in fungal rhinosinusitis'), along with the fact that biopsy procedures are time-consuming and often not feasible due to the moribund status of the patient (Dhiwakar et al 2003), amphotericin may be started empirically if clinical suspicion warrants so.

Intraorbital amphotericin: There are numerous references to suggest the role of intraorbital Amphotericin B. Seiff et al (1999) have stated that conservative orbital debridement with local Amphotericin B irrigations is an effective adjunct in the control of sino-orbital fungal infections, especially in patients with reversible immunosuppression and good preoperative visual acuities. ${ }^{17}$ The use of the drug has also been described for the successful palliative treatment of an Aspergillus fumigatus orbital mass in a patient with AIDS by direct injection of Amphotericin B into the abscess cavity. ${ }^{18}$ Similar reports have emerged from Kahana and Lucarelli (2007), who have also gone on to describe the technique for the same using a bariumembedded neurosurgical catheter for intraorbital drug delivery. ${ }^{19}$ Wakabayashi et al (2007) have described retrobulbar injections for the treatment of invasive sino-orbital Aspergillosis. $^{20}$

\section{THE AZOLES}

The azoles are a group of drugs which are gaining increasing popularity for their effectiveness against fungal rhinosinusitis. Of these, fluconazole and itraconazole are the most widely used. They both belong to the triazole family.

These drugs act by inhibiting the cytochrome P-450 dependent enzyme lanosterol 14-alpha-demethylase, which is necessary for the conversion of lanosterol to ergosterol, ${ }^{14,21}$ which is a vital component of the fungal cell membrane.

The common side effects are rashes, headaches and gastrointestinal upsets and mild elevations in liver function tests. Higher doses of itraconazole have been associated with an aldosterone-like effects (Luna et al 2000).

The doses range from 100 to $400 \mathrm{mg}$ per day in two divided doses. Itraconazole has been used for indolent, nonmeningeal Aspergillosis in doses of $400 \mathrm{mg} / \mathrm{day} .{ }^{22}$ Itraconazole has the best activity against fungi such as Aspergillus, and black moulds, that typically cause fungal sinusitis, and is probably the drug of choice for this site of infection unless there is extensive invasion into bone, in which case initial therapy with amphotericin B may be necessary. ${ }^{15}$ It is an inappropriate agent for rhinocerebral mucormycoses, which requires Amphotericin B or its lipid preparations. ${ }^{15}$

There have been reports of the efficacy of itraconazole for the treatment of AFRS and fungal balls (vide supra), but it probably needs more trials to validate this.

\section{Amphotericin B and Itraconazole-antagonists}

Numerous reports have shown the antagonistic effects between amphotericin B and triazoles such as itraconazole and fluconazole. ${ }^{23,24}$ Similar animal models have exhibited either antagonism or no change efficacy. ${ }^{25}$ However, on the other hand, clinical experience has not generally demonstrated azole-polyene antagonism. This can be seen in cases of many deep seated severe mycoses, which are initially treated with amphotericin B and subsequently a triazole is added. ${ }^{15}$ This potential antagonism can probably be explained on the common link of ergosterol; wherein triazoles prevent formation of ergosterol in the cell membrane, polyenes bind to it and increase cellular permeability.

\section{ADJUVANT THERAPY}

Hyperbaric oxygen therapy (HBOT): It is known to increase tissue oxygen levels, boost leucocyte killing capacity, and may have an additive effect with Amphotericin $\mathrm{B}$ in rhino-orbito-cerebral mucormycoses. ${ }^{30}$ It is known to alleviate acidosis and improve neutrophilic phagocytosing capacity, which is extremely important to fight mucormycosis. ${ }^{32}$ It has also been shown to inhibit the germination of fungal spores and the growth of the mycelia in vitro. ${ }^{31}$ 
Granulocyte colony stimulating factor (GCSF): This boosts polymorphonuclear fungicidal activity; however, whether this translates into an improved clinical outcome, particularly in those who are not granulocytopenic is unknown. ${ }^{30}$ However, in neutropenic patients with invasive fungal sinusitis, this is certainly helpful.

\section{THE FUTURE}

Voriconazole: A derivative of fluconazole and a triazole by itself, it is gaining increasing acceptance against acute and chronic invasive Aspergillosis, as well as various forms of Candidiasis. Commonly administered in an initial loading dose of intravenous voriconazole, $6 \mathrm{mg} / \mathrm{kg}$ every 12 hours for 2 doses, followed by $3 \mathrm{mg} / \mathrm{kg}$ every 12 hours for the next 30 days. This may be followed by oral voriconazole $200 \mathrm{mg} 12$ hourly to complete 24 weeks totally. ${ }^{26}$ The common side effects are transient visual disturbances and mild elevation of liver enzymes. The only limiting factor is the cost of the drug.

Posaconazole: Another azole, active against a variety of yeasts and moulds including zygomycetes. ${ }^{27}$ It may be used as a salvage therapy for a variety of invasive fungal infections after the failure or intolerance to other antifungals. ${ }^{28}$ It has also been proven to be of use in cases of rhino-orbital zygomycosis caused by Apohysomyces elegans in a immunocompetent host. ${ }^{29}$

\section{Caspofungin}

This is another newer drug, of the enichocandins class of drugs, which has been recently introduced for the empirical management of neutropenic patients suspected of having fungal sinusitis. It has proven to be cost effective as compared to liposomal Amphotericin B in the management of fungal infections.

\section{REFERENCES}

1. Kupferberg SB, Bent JP III, Kuhn FA. Prognosis for Allergic Fungal Sinusitis. Otolaryngol Head Neck Surg 1997;117:35-41.

2. Kuhn FA, Javer AR. Allergic Fungal Sinusitis: A four year followup. Am J Rhinol 2000;14:149-56.

3. Stevens DA, Schwartz HJ, Lee JY, et al. A randomised trial of itraconazole in allergic bronchopulmonary Aspergillosis. N Engl J Med 2000;342:756-62.

4. Wark PABW, Hensley MJ, Saltos N, et al. Anti-inflammatory effect of itraconazole in stable bronchopulmonary Aspergillosis: A randomised controlled trial. J Allergy Clin Immunol 2003;111:952-57.
5. Schubert MS. Medical Treatment of Allergic Fungal Sinusitis. Ann Allergy Asthma Immunol 2000;85:90-101.

6. Kupferberg SB, Bent JP III. Allergic fungal sinusitis in the paediatric population. Arch Otolaryngol Head Neck Surg 1996;122:1381-84.

7. Sher TH, Schwartz HJ. Allergic Aspergillus Sinusitis with concurrent allergic bronchopulmonary Aspergillosis: Report of a case. J Allergy Clin Immunol 1988;81:844-46.

8. Adelson RT, Marple BF. Fungal rhinosinusitis - state-of-theart diagnosis and treatment . J Otolaryngol 2005;34(Suppl):S18S22.

9. Klossek JM, Serrano E, Peliquin L, Percodani J, Fontanel JP, Pessey JJ. Functional endoscopic sinus surgery and 109 mycetomas of the paranasal sinuses. Laryngoscope 1997;107:112-17.

10. Ferguson BJ. Fungal Balls of the Paranasal Sinuses. Otolaryngol Clin N Am 2000;33:398-98.

11. Rains BM 3rd, Mineck CW. Treatment of allergic fungal sinusitis with high dose itraconazole. Am J Rhinol 2003;17:1-8.

12. Andersen CM. Sodium Chloride treatment of amphotericin B nephrotoxicity: Standard of care. West J Med 1995;162:313-17.

13. Gallis HA, Drew RH, Pickard WW. Amphoterecin B: 30 years of clinical experience. Reviews of Infectious Diseases 1990;12:308-29.

14. Groll AH, Piscitelli SC, Walsh TJ. Clinical Pharmacology of systemic antifungal agents in clinical use, current ivestgational compounds and putative targets for antifungal drug development. Adv Pharmacol 1998;44:343-501.

15. Luna B, Drew RH, Perfect JR. Agents for treatment of invasive fungal infections. Otolaryngol Clin N Am 2000;33(2):277-99).

16. Kauffman CA, Carver PL. Antifungal agents in the 1990's: current status and future developments. Drugs 1997;53:539-49.

17. Seiff SR, Choo PH, Carter SR. Role of local amphotericin B therapy for sino-orbital fungal infections. Ophthal Plast Reconstr Surg 1999;15(1):28-31.

18. Cahill KV, Hogan CD, Koletar SL, Gersman M. Intraorbital injection of amphotericin B for palliative treatment of Aspergillus orbital abscess. Ophthal Plast Reconstr Surg 1994;10(4):27677.

19. Kahana A, Lucarelli MJ. Use of Radio-opaque Intraorbital catheter in the treatment of Sino-Orbital-Cranial Mucormycosis. Arch Ophthalmol 2007;125(12).

20. Wakabayashi T, Oda H, Kinoshita N, Ogasawara A, Fujishiro Y, Kawanabe W. Retrobulbar amphotericin B injections for treatment of invasive sino-orbital Aspergillosis. Jpn J Ophthalmol 2007;51(4):309-11.

21. Kauffman CA, Carver PL. Use of azoles for systemic antifungal therapy. Adv Pharmacol 1997;39:143-89.

22. De Beule K, De Doncker P, Cauwenbergh G. The treatment of Aspergillosis and aspergilloma with itraconazole, clinical results of an open international study (1982-1987). Mycoses 1988;31:476-85.

23. Ghannoum MA, Fu Y, Ibrahim AS, et al. In vitro determination of optimal antifungal combinations against Cryptococcus neoformans and Candida albicans. Antimicrob Agents Chemother 1995;39:2459-65.

24. Lewis RE, Lund BC, Klepser ME. Assessment of the antifungal activity of fluconazole and amphotericin B administered alone, 
and in combination against Candida albicans by using a dynamic in vitro mycotic model. Antimicrob Agents Chemother 1998;42:1382-86.

25. Schmitt H, Berbard EM, Edwards FF. Combination therapy in a model of pulmonary Aspergillosis. Mycoses 1991;34:281-85.

26. Sheehan DJ, Hitchcock CA, Sibley CM. Current and emerging azole antifungal agents. Clin Microbiol Rev 1999;12:40-79.

27. Torres HA, Hachem RY, Chemaly RF, Kontoyiannis DP, Raad II. Posaconazole: A broad-spectrum triazole antifungal. Lancet Infect Dis 2005;5:775-85.

28. van Burik JA, Hare RS, Solomon HF, Corrado ML, Kontoyiannis DP. Posaconazole is effective as salvage therapy in zygomycosis: A retrospective summary of 91 cases. Clin Infect Dis 2006;42:c61-c65.
29. Ferguson TD, Schneiderjan SD, Dionne-Odom J, Brandt ME, Rinaldi MG, Nolte FS, Langston A, Zimmer SM. Posaconazole treatment for Apohysomyces elegans rhino-orbital zygomycosis following trauma for a male with well-controlled diabetes. Journal of Clinical microbiology 2007;45(5):1648-51.

30. Liang KP, Tleyjeh IM, Wilson WR, Roberts GD, Temesgen Z. Rhino-orbitocerebral mucormycoses caused by Apohysomyces elegans. J Clin Microbiol 2006;44(3):892-98.

31. Spellberg B, Edwards J, Ibrahim A. Novel perspectives on mucormysoses: Pathophysiology, presentation and management. Clin Microbiol Rev 2005;18:556-69.

32. Couch L, Theilen F, Mader JT. Rhincerebral mucormycosis with cerebral extension successfully treated with adjunctive hyperaric oxygen therapy. Arch Otolaryngol Head Neck Surg 1988;114:791-94. 